\title{
Uveitis in Juvenile Chronic Arthritis: Incidence, Clinical Features and Prognosis
}

\author{
JACK J. KANSKI
}

Windsor

\begin{abstract}
Summary
Three hundred and fifteen patients with anterior uveitis and juvenile chronic arthritis were reviewed in order to determine the incidence, visual prognosis, and the clinical characteristics of the intraocular inflammation. The overall incidence of uveitis was $20 \%$. Approximately $25 \%$ of patients had relatively mild and/or transient involvement and an excellent visual prognosis. In $50 \%$ the uveitis was more severe but could be controlled with topical medication. In the remaining $25 \%$ the visual prognosis was poor due to the intractable nature of the uveitis and the subsequent development of vision-threatening complications. The majority of patients (74\%) were under the age of 8 years when the uveitis was first diagnosed. Clinically, the intraocular inflammation was most frequently an asymptomatic, chronic, non-granulomatous, iridocyclitis which was bilateral in $71 \%$ of cases. Other ocular lesions, which were rare, included keratoconjunctivitis sicca and corneal melting.
\end{abstract}

Juvenile chronic arthritis (JCA) is defined as arthritis affecting one or more joints for at least 3 months in patients under the age of 16 years, other causes of arthropathy which may mimic the condition (e.g. systemic lupus erythematosus, trauma, infection, rheumatic fever etc.) having been excluded. JCA was formerly called Still's disease and in the United States it is frequently referred to as juvenile 'rheumatoid' arthritis. The association between anterior uveitis and JCA is now well established and was first reported by $\mathrm{Ohm}$ in $1910 .{ }^{1}$ According to the mode of onset, the three subgroups of JCA are pauciarticular, polyarticular, and systemic Although it is agreed that young girls with pauciarticular onset JCA who are also positive for circulating antinuclear antibodies carry the highest risk of uveitis and those with systemic onset JCA the least risk, ${ }^{2-5}$ the literature contains conflicting reports concerning the incidence of uveitis (between $2 \%$ to $21 \%$ ).$^{2,6-14}$ Because JCA is a relatively rare condition, very few ophthalmologists, even those with a special interest in uveitis, have the opportunity of managing a large number of cases and it has been the author's impression that many feel that the visual prognosis is universally poor and some have even doubted the value of any type of therapy. ${ }^{15-16}$ The main purpose of this report is to describe a large number of patients with JCA and uveitis followed over a long period of time in order to determine more accurately the incidence of uveitis and to show that in the majority of patients the visual prognosis is relatively good provided the intraocular inflammation is detected and treated early. The clinical characteristics of the uveitis as well as other ocular lesions are also described.

From: Juvenile Rheumatism Unit, Canadian Red Cross Memorial Hospital, Taplow and Division of Rheumatology, Clinical Research Centre, Northwick Park Hospital, Harrow.

Part of the thesis of MD, University of London.

Correspondence to: J. J. Kanski, FRCS, Bay Lodge, Woodhill Avenue, Gerrards Cross, Bucks, SL9 8DP. 


\section{Materials and methods}

The incidence of uveitis in JCA was determined by studying 122 patients with JCA all of whom fulfilled the following criteria:

(a) they were followed up for at least 5 years

(b) they were initially evaluated within three months of the onset of arthritis,

(c) they had regular slitlamp biomicroscopy by the author or one of his assistants when they attended the combined 'joint eye' clinic.

In order to evaluate the visual prognosis, a total of 103 patients with JCA and uveitis were studied. All patients fulfilled the following criteria:

(a) they had a minimal follow up of two years from the time of diagnosis of uveitis; the mean follow-up period was 7 years 3 months (range 2 to 14 years),

(b) the uveitis was initially diagnosed prior to the development of complications (i.e. posterior synechiae, band keratopathy, secondary inflammatory glaucoma, and complicated cataract),

(c) they were all personally examined and treated by the author although some patients seen prior to January 1975 were initially managed by the author's predecessor.

In order to establish the clinical characteristics of uveitis the medical records of 315 patients with JCA and uveitis were reviewed. The vast majority had been examined by the author personally during a ten year period (January 1975 to December 1984), either at the Juvenile Rheumatism Unit, Canadian Red Cross Memorial Hospital, Taplow, or at the Division of Rheumatology, Clinical Research Centre, Northwick Park Hospital, Harrow. Apart from routine slitlamp biomicroscopy, applanation tonometry, and examination of the fundi with direct and indirect ophthalmoscopy, special investigations to detect the possibility of asymptomatic complications unrelated to uveitis were not performed (e.g. staining of the conjunctiva with rose bengal).

\section{Results}

\section{(a) Incidence of uveitis.}

Twenty five patients out of $122(20 \%)$ of the patients developed uveitis.

\section{(b) Visual prognosis}

Although there was some overlap, it was possible to divide the 103 patients into four groups according to the severity and duration of intraocular inflammation as well as the presence or absence of vision-threatening complications.

Group 1. The seven patients in this group had very mild uveitis without keratic precipitates and never more than +1 cells in the aqueous humour. In only one patient did the intraocular inflammation persist for longer than 12 months. None of the patients were treated with topical corticosteroids as it was felt that the risk of steroid-induced complications (glaucoma and cataract) was greater than the risk of vision-threatening complications due to uveitis.

Group 2. The seventeen patients in this group had one attack of uveitis which lasted less than 4 months. The severity of inflammation varied from +2 to +4 cells. In all patients the uveitis was controlled with topical dexamethasone drops. All patients were also treated with twice daily instillation of tropicamide $1 \%$ drops. When last examined none of the patients had developed posterior synechiae, band keratopathy, secondary inflammatory glaucoma, or complicated cataract.

Group 3. The fifty-two patients in this group had moderate to severe ( +2 to -4 cells) uveitis which persisted for more than 4 months. The majority had acute exacerbations of intraocular inflammation which usually could be controlled by a three day course of very frequent (hourly or two-hourly) instillation of dexamethasone drops. Three patients who were unresponsive to topical medication were treated by anterior subTenon's injections of methylprednisolone (Depomedrone). When last examined most patients were still on topical medication, four had developed visually inconsequential lens opacities and one patient had lens opacities as well as secondary inflammatory glaucoma.

Group 4. All 27 patients in this group had severe ( +3 to +4 cells) and prolonged uveitis 
which responded poorly to topical corticosteroid medication. Because of the intractable nature of the intraocular inflammation and the fear of blindness, three patients were treated with systemic chlorambucil. When last examined, all patients in this group had had cataract surgery in at least one eye, and thirteen patients also had secondary inflammatory glaucoma.

In this entire group of 103 patients, the incidence of complicated cataract was $31 \%$ (32 patients) and 14\% (14 patients) had secondary inflammatory glaucoma.

\section{(c) Clinical features of uveitis}

(i) Bilateral involvement. Seventy one per cent (233 of 315$)$ of patients had bilateral uveitis. In most cases both eyes were either involved simultaneously or within a few months of each other. It was unusual for patients with initially unilateral uveitis to develop involvement of the second eye after more than one year. In those with bilateral uveitis, the severity of intraocular inflammation was usually symmetrical.

(ii) Age at diagnosis of uveitis. Because of the frequently asymptomatic nature of the intraocular inflammation it was not possible to give a precise age of the patients at the time of its commencement. For this reason only the age at which the uveitis was initially detected is given. The mean age was 6 years (range 1 to 35 years), and $74 \%$ (233) of the patients were under the age of 8 years when the uveitis was initially diagnosed.

(iii) Symptoms. The onset of uveitis was invariably asymptomatic and detected on routine slitlamp biomicroscopy. Even during acute exacerbations with +4 cells in the aqueous humour it was rare for patients to complain, although a few older children noticed an increase in floaters.

(iv) External signs. Acute exacerbations are not associated with a ciliary flush.

(v) Slitlamp signs. Keratic precipitates were usually small to medium in size and were located mainly in the inferior half of the cornea. A few patients had large keratic precipitates which in one case were of the 'mutton fat' variety. Hypopyon was seen in only two patients during acute and severe exacerbations. Posterior synechiae were common in eyes with longstanding neglected uveitis and in some cases the iris surface contained dilated blood vessels which extended onto the lens. Keoppe iris nodules were seen in a few cases. In eyes with moderate to severe uveitis, cells in the anterior vitreous were frequent.

(vi) Posterior segment signs. Cystoid macular oedema was rare in eyes that had not undergone cataract surgery.

\section{(d) Other ocular lesions}

Apart from uveitis, other ocular lesions were found in nine patients. Five had keratoconjunctivitis sicca characterised by corneal filaments and the typical staining pattern of the conjunctiva with rose bengal. Surprisingly, none of the patients had symptoms and for this reason treatment with tear substitutes was felt to be unnecessary. In three eyes of two patients inactive chorioretinal scars were seen at the posterior pole. One patient developed severe unilateral corneal thinning which was not associated with scleritis. The eye was subsequently enucleated as the cornea had perforated. Another patient developed transient unilateral inferior superficial corneal stromal infiltrates.

\section{Discussion and Conclusions}

The true incidence of uveitis in patients with JCA is dependent on the length of follow up and the care with which the eyes are examined. The incidence of $20 \%$ in this report is still probably a slight underestimation, because a few patients develop uveitis many years after the onset of arthritis. The incidence of uveitis in other series varies from $2 \%$ to $21 \% .^{2,6-14}$ This discrepancy is probably due to variations in the length of follow up and the fact that in some reports the patients were initially examined by nonophthalmologists and no particular attention was paid to the eyes of asymptomatic patients.

It is evident that, as in the case of joint involvement, the intraocular inflammation can be subdivided into several groups according to severity and duration. It is, however, only after a prolonged follow up that the 
characteristics of these groups can be delineated accurately. It is certainly true that until the advent of cortisone the only available treatment for uveitis was with mydriatics and non-specific agents which had no proven anti-inflammatory action, and until Blegvad in $1941^{17}$ reported the first case of JCA with uveitis unassociated with band keratopathy it was generally assumed that most patients eventually became blind. Smiley (1974) ${ }^{18}$ felt that although in the majority of patients the visual prognosis was poor, there were a few with relatively mild involvement and a good prognosis. He stated that the facts determining this benign course in some patients were still unknown, treatment alone was not the only explanation since many children who had received identical therapy over a similar period of time had slowly deteriorated. It is, however, important to emphasise that during the fifties and early sixties, when Smiley treated most of his patients, only relatively weak topical corticosteroids preparations such as cortisone and hydrocortisone were available in contrast to the potent corticosteroids which are now at our disposal such as dexamethasone, betamethasone, and prednisone.

This study has shown that in about $25 \%$ of cases the prognosis is very good and the uveitis either requires no specific treatment or responds readily to topical corticosteroids. At the other end of the spectrum are patients with very severe and prolonged inflammation which respond poorly to treatment. This group accounts for about $25 \%$ of cases and the vast majority of patients eventually require surgery for complicated cataract and/ or secondary glaucoma. The largest group, accounting for about $50 \%$ of patients, is characterised by moderate to severe uveitis which responds reasonably well to topical corticosteroids although treatment may have to be continued for months and even years. The long term visual prognosis is good although a few eventually develop complications.

The intraocular inflammation in patients with JCA was typically an insidious, asymptomatic, chronic iridocyclitis. Because of the initially asymptomatic nature of the uveitis it is extremely important to screen patients at regular intervals so that the condition can be treated prior to the development of complications. Although a few patients had Koeppe nodules and one had 'mutton fat' keratic precipitates, the uveitis was nongranulomatous in the majority of cases. The frequent incidence of bilateral involvement of $71 \%$ is in the general agreement with other reports of between $67 \%$ and $89 \% .10,11,14,18,19$ Of more practical interest to the ophthalmologist and parents is the finding that it was unusual for children with initially unilateral uveitis to develop involvement of the second eye after a period of more than twelve months.

Although no specific tests were routinely performed to detect keratoconjunctivitis sicca, there were five patients with dry eyes. Chylack and co-workers ${ }^{2}$ claim to be the first to report keratoconjunctivitis sicca in association with JCA and uveitis. All three of their patients had symptoms in contrast to the five patients in this study who were all asymptomatic. As far as is known, this is the first report of peripheral corneal infiltrates and corneal thinning in patients with uveitis and JCA. The significance of transient limbal infiltrates in one patient is difficult to interpret although it is tempting to speculate that the severe peripheral corneal thinning which eventually led to perforation and enucleation in the other patients was related to JCA. However, the finding of old chorioretinal scars in the other two patients is almost certainly coincidental.

In adults there appears to be very little overlap between the ocular complications of sero-positive (for rheumatoid factor) rheumatoid arthritis and the sero-negative arthritides (e.g. ankylosing spondylitis, Reiter's syndrome etc.). In rheumatoid arthritis, the ocular lesions are invariably confined to the outer eye (keratoconjunctivitis sicca, keratitis and scleritis) and the incidence of uveitis is no different than in the general population. In contrast the main ocular complication of the sero-negative arthritides is anterior uveitis. It is therefore surprising that five patients with JCA, which is a sero-negative arthritis, developed keratoconjunctivitis sicca and another keratitis. This finding suggests that in children the overlap between 
the ocular manifestations of the sero-positive and sero-negative may be more common than in adults.

\section{References}

${ }^{1} \mathrm{Ohm} \mathrm{J}$ : Bandformige Hornhauttrubung bei einen neunjahrigen Madchen und ihre Behandlung mit subkonjunktivalen Jodkaliumeinspritzungen. Klin Mbl Augenheilk 1910, 48; 243-45.

${ }^{2}$ Chylack LT Jr, Bienfang DC, Bellows AR, Stillman JS: Ocular manifestations of juvenile rheumatoid arthritis. Am J Ophthalmol, 1975; 79: 1026-33.

${ }^{3}$ Ohno S, Char DH, Kimura SJ, O'Connor GR: HLA antigens and antinuclear antibody titres in juvenile chronic iridocyclitis. Br J Ophthalmol, 1977, 61: 59-61.

${ }^{4}$ Kanski JJ and Shun-Shin GA: Systemic uveitis syndromes in childhood: an analysis of 340 cases. Ophthalmology, 1984; 91: 1247-52.

${ }^{5}$ Schaller JG, Johnson GD, . Holborow EJ, Ansell BM, Smiley WK: The association of antinuclear antibodies with the chronic iridocyclitis of juvenile rheumatoid arthritis (Still's disease). Arthritis Rheum, 1974, 17: 409-16.

${ }^{6}$ Vesterdal E and Sury B: Iridocyclitis and bandshaped corneal opacity in juvenile rheumatoid arthritis. Acta Ophthalmol, 1950; 28: 321-37.

${ }^{7}$ Smiley WK, May E, Bywaters EGL: Ocular presentations of Still's disease: Its complications and treatment. Ann Rheum Dis, 1957; 16: 371-83.
${ }^{8}$ Edstrom G: Band-shaped keratitis in juvenile rheumatoid arthritis. Acta Rheumatol Scan, 1961; 7: 169-73.

${ }^{9}$ Smiley WK: Iridocyclitis in Still's disease. Trans Ophthalmol Soc UK, 1965; 85: 351-6.

${ }^{10}$ Stewart AJ and Hill BM: Ocular manifestations in juvenile rheumatoid arthritis. Can $J$ Ophthalmol, 1967; 2: 58-62.

${ }^{11}$ Kazdan JJ, McCulloch JC, Crawford JS: Uveitis in children. J Ass Med Can, 1967; 96: 385-91.

${ }^{12}$ Schaller $\mathrm{J}$ and Wedgwood RJ: Juvenile rheumatoid arthritis: A review. Pediatrics, 1972, 50: 940-53.

${ }^{13}$ Goel, KM and Shanks RA: Follow-up study of 100 cases of juvenile rheumatoid arthritis. Ann Rheum Dis, 1974; 33: 25-31.

${ }^{14}$ Spalter HF: The visual prognosis in juvenile rheumatoid arthritis. Trans Am Ophthalmol Soc, 1975; 3: 554-7.

15 Tressler H: Uveitis. In Peyman GA, Sanders DR, Goldberg MF eds. Principles and practice of ophthalmology. Philadelphia. WB Saunders 1980: 1581.

${ }^{16}$ Smith RE and Nozik RM: Uveitis. A clinical approach to diagnosis and management. Baltimore. Williams and Wilkins 1983: 156.

${ }^{17}$ Blegvag O: Iridocyclitis and disease of the joints of children. Acta Ophthalmol, 1941; 19: 21936.

${ }^{18}$ Smiley WK: The eye in juvenile rheumatoid arthritis. Trans Ophthalmol Soc UK, 1974; 94: 817-29.

${ }^{19}$ Key SN and Kimura SJ: Iridocyclitis associated with juvenile rheumatoid arthritis. Am J Ophthalmol, 1975; 80: 425-29. 